\title{
Measurements at laser materials processing machines: spectrum deconvolution including uncertainties and model selection
}

\author{
Rolf Behrens $^{1}$, Björn Pullner ${ }^{1}$, and Marcel Reginatto ${ }^{2}$ \\ ${ }^{1}$ Department Radiation Protection Dosimetry, Physikalisch-Technische Bundesanstalt (PTB), \\ Bundesallee 100, 38116 Braunschweig, Germany \\ ${ }^{2}$ Department Neutron Radiation, Physikalisch-Technische Bundesanstalt (PTB), \\ Bundesallee 100, 38116 Braunschweig, Germany \\ Correspondence: Rolf Behrens (rolf.behrens@ptb.de)
}

Received: 7 September 2020 - Revised: 9 January 2021 - Accepted: 12 January 2021 - Published: 18 February 2021

\begin{abstract}
Laser materials processing of workpieces using ultra-short pulsed lasers can lead to unwanted X-ray emission. Their dose rate and spectral distribution have been precisely determined. The measurements were carried out using a thermoluminescence detector (TLD)-based spectrometer in which 30 TLD planes are arranged one behind the other, the first 10 layers made of polymethyl methacrylate, while the remaining 20 layers are interspaced by absorbers with, from the front to the back, increasing atomic charge and thickness. The penetration depth of the radiation into the spectrometer depends on its energy, so that the energy-resolved spectrum of the radiation can be calculated from the TLD dose values by means of mathematical methods (Bayesian deconvolution). The evaluation process also takes into account both the uncertainties of all input quantities and the possibility of adopting different models for the spectrum form. This allowed the resulting spectra to be associated with their realistic uncertainty.

The measurements are traceable to the Système international d'unités (SI), i.e. the International System of Units. The results not only provide manufacturers and users of ultra-short pulsed lasers with important information on the design of the machines with regard to radiation protection, but were also included in the recently concluded legislative procedure in the field of radiation protection in Germany.
\end{abstract}

\section{Background, motivation, and objective}

Ultra-short pulsed lasers have been developed in the last decades for both research and various applications (Mulser and Bauer, 2010). In some of these applications, the main purpose is the production of ionizing radiation, with laser intensities at the focus of the order of up to $10^{22} \mathrm{~W} / \mathrm{cm}^{2}$. Much lower intensities have been in use for materials processing without the production of ionizing radiation (Momma et al., 1996). However, in the last few years, larger intensities of up to the order of $10^{14} \mathrm{~W} / \mathrm{cm}^{2}$ have been in use for materials processing, resulting in an unwanted production of ionizing radiation (Gerhard et al., 2016; Nolte et al., 2016). The objective of this research is twofold, the validation of the spectrometer in the presence of photons in the low-energy range and the measurement of X-rays emitted in an industrial laser materials processing environment in terms of absolute spectra and operational dose equivalent with the focus on radiation protection. At the present time, this objective is more important from a research point of view as current industrial laser materials processing machines are equipped under normal conditions with a housing that is very effective for shielding the low-energy radiation emerging from the machines. The results are relevant to developers of the machines also operating them without housing, to legislative bodies, and as a basis for future investigations of radiation from laser machines with even higher intensities. 


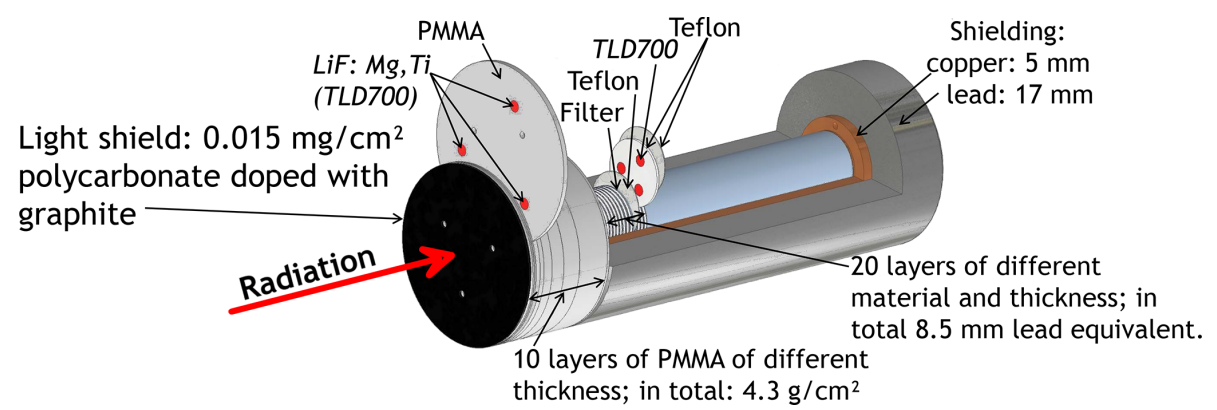

Figure 1. Sketch of the TLD-based spectrometer. Basic principle: the deeper the radiation penetrates the spectrometer, the higher its energy.
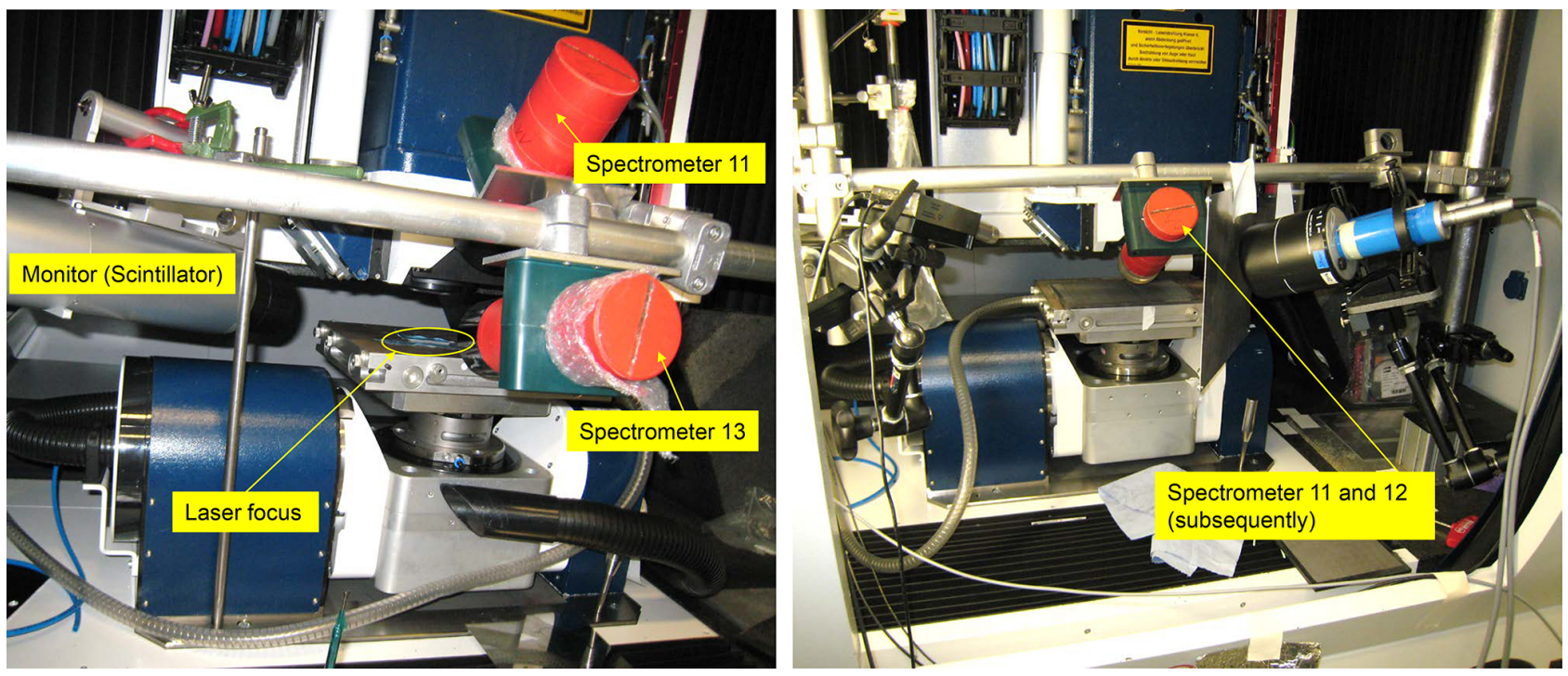

Figure 2. Experimental setup of the two performed measurement series.

\section{Measurements and data evaluation}

For the measurements, a thermoluminescence detector (TLD)-based few-channel spectrometer was used (see Fig. 1) (Behrens and Ambrosi, 2002; Behrens, 2009). The penetration depth of the X-ray radiation in the spectrometer depends on the energy, so that the energy-resolved and absolute spectrum of the radiation, including the uncertainties of the spectrum, can be determined from the dose values in the TLD layers by means of mathematical methods (Bayesian deconvolution).

The experimental setup is shown in Fig. 2; the main laser parameters were a wavelength of $1030 \mathrm{~nm}$, an average power of $78 \mathrm{~W}$, a pulse energy of $195 \mu \mathrm{J}$, a pulse length (full width at half maximum) of $924 \mathrm{fs}$, a repetition rate of $400 \mathrm{kHz}$, and a focus diameter of $16 \mu \mathrm{m}$ at a focus intensity of $2.1 \times 10^{14} \mathrm{~W} / \mathrm{cm}^{2}$; further experimental parameters are listed in Table 1. A Bayesian data evaluation was performed using the WinBUGS software (Lunn et al., 2010) which, besides the photon spectrum, also supplies the corresponding uncertainties and coverage intervals. The following prior in- formation for the photon spectra was included in the data evaluation: (i) a smooth rise with increasing energy due to the fact that there was at least about $10 \mathrm{~cm}$ of air absorption between the laser focus and the spectrometer front; (ii) an exponential decrease at higher energies (due to well-known laser-plasma interaction mechanisms); and (iii) a peak in the spectrum at the energy of the characteristic fluorescence radiation of the workpiece material. The method of analysis is outlined in detail in Appendix A as well as in the literature (Behrens et al., 2019; Behrens and Reginatto, 2019).

\section{Validation of the data evaluation}

To ensure that the evaluation method is reliable, the spectrometer was irradiated in four known reference radiation fields of different photon energies. Figure 3 shows the spectra from the corresponding data evaluation compared to the results measured with a high-purity germanium spectrometer (Ankerhold et al., 1999; Ankerhold, 2000). The radiation fields are specified in an ISO standard (ISO, 2019). 

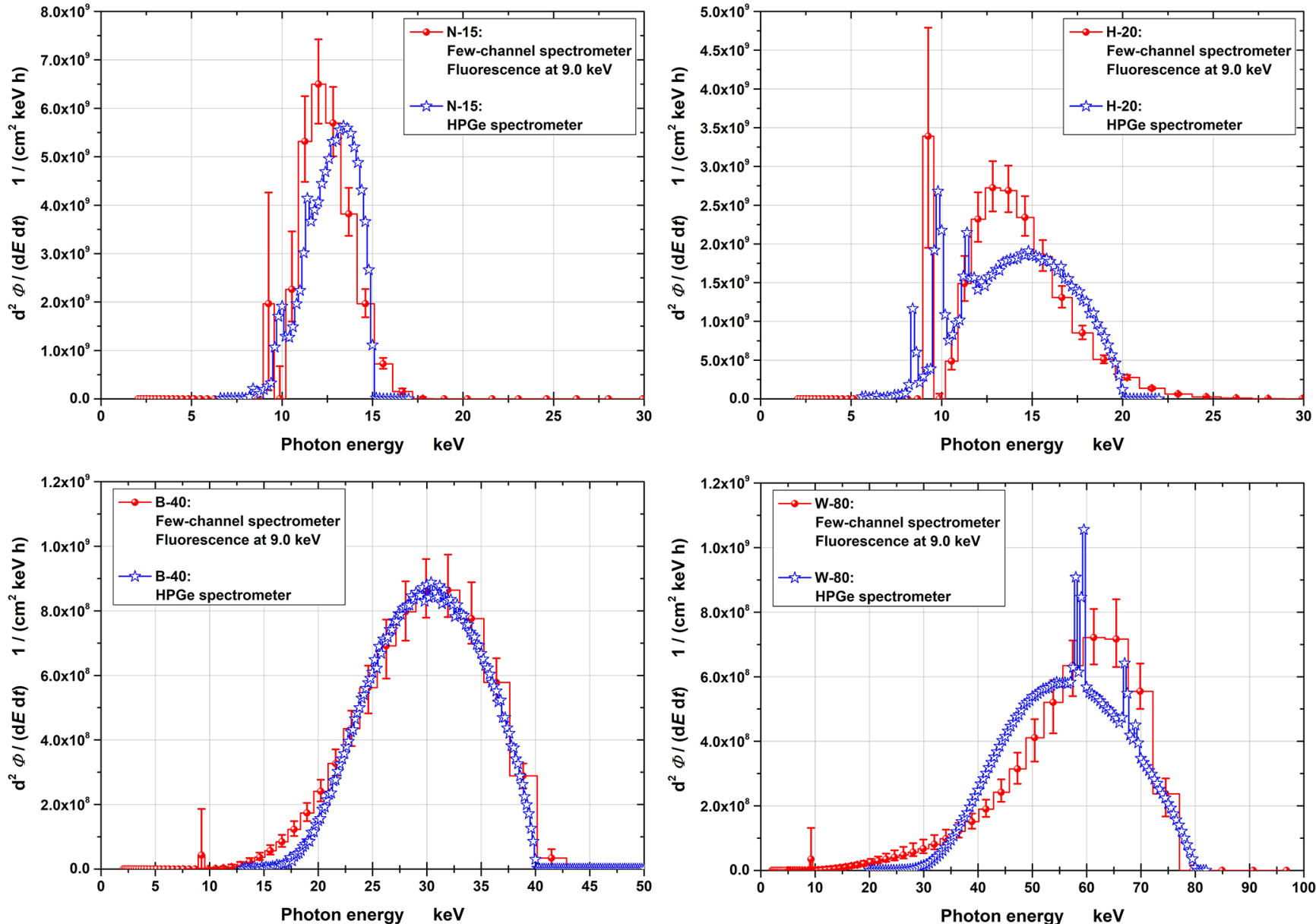

Figure 3. Comparison of the spectra measured with the few-channel spectrometer (red line) and those measured with the high-purity germanium spectrometer (blue lines). Meaning of the uncertainty bars: the value of the spectral fluence is usually located with a probability of approximately $95 \%$ in the assigned coverage interval.

Table 1. Details of the experiments.

\begin{tabular}{|c|c|c|c|c|}
\hline \multirow[t]{2}{*}{ Parameter } & \multicolumn{2}{|c|}{ First setup } & \multicolumn{2}{|c|}{ Second setup } \\
\hline & Spectrometer 11 & Spectrometer 13 & Spectrometer 11 & Spectrometer 12 \\
\hline Workpiece material & Tungsten & & Steel (St37) & Alloy* \\
\hline Workpiece thickness & $0.2 \mathrm{~mm}$ & & $6 \mathrm{~mm}$ & \\
\hline Workpiece condition & $\begin{array}{l}\text { Plane parallelism } \\
\text { specified }\end{array}$ & Ind flatness not & $\begin{array}{l}\text { Plane parallelism } \\
\text { approximately } 15\end{array}$ & im flatness \\
\hline $\begin{array}{l}\text { Frequency of workpiece processing } \\
\text { at the same surface position }\end{array}$ & One time & & Up to seven times & \\
\hline Angle relative to workpiece surface & $46^{\circ}$ & $13^{\circ}$ & $31^{\circ}$ & \\
\hline $\begin{array}{l}\text { Distance } x \text { of the spectrometer front } \\
\text { from the laser focus }\end{array}$ & $17 \mathrm{~cm}$ & & $9.76 \mathrm{~cm}$ & \\
\hline Effective irradiation time & $2.6 \mathrm{~h}$ & & $3.1 \mathrm{~h}$ & $2.2 \mathrm{~h}$ \\
\hline
\end{tabular}

* $92.5 \%$ mass fraction tungsten; $3.75 \%$ mass fraction iron; $3.75 \%$ mass fraction nickel. 


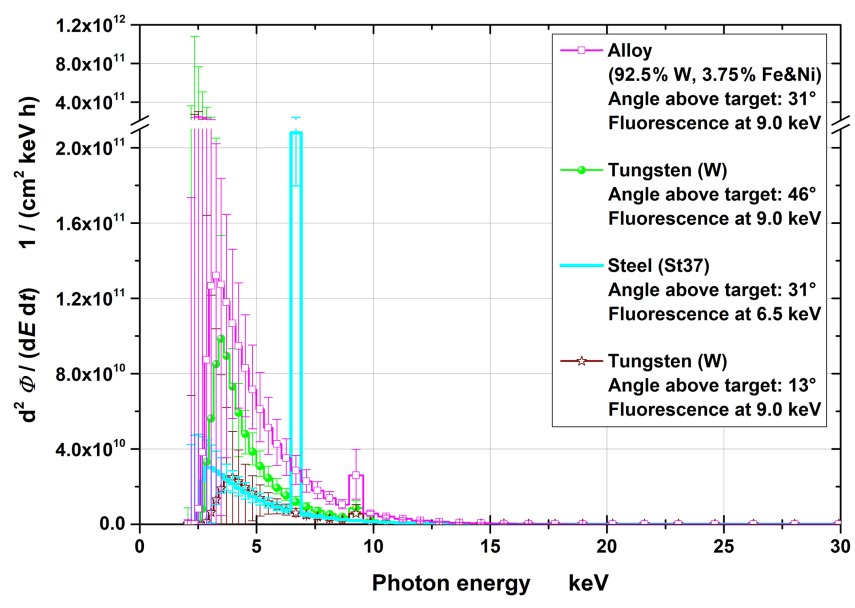

Figure 4. Photon fluence spectra (normalized to $1 \mathrm{~h}$ effective irradiation time and $10 \mathrm{~cm}$ distance) together with their $95 \%$ coverage intervals. Note the broken ordinate.

The $95 \%$ coverage intervals were determined from the probability distribution for each energy channel, which is calculated as part of the Bayesian data evaluation. Thus, they incorporate the information about the measurement used for the data evaluation, i.e. the prior information described in Sect. 2.

Apart from a slight shift in the spectra's maximum, these are quite well reproduced with the few-channel spectrometer as compared to the spectra obtained using the germanium spectrometer. The shift is mainly present when significant characteristic lines from the X-ray anode material are contained in the spectrum - indicating the limits of the few-channel spectrometer's energy resolution. Furthermore, the absolute magnitude of the spectra is in good agreement, demonstrating the capability for an absolute dose determination.

\section{Results}

Figure 4 shows the absolute photon fluence spectra per materials processing time together with their $95 \%$ coverage intervals for the four measurements normalized to a distance of $10 \mathrm{~cm}$ from the workpiece. The mean energies vary from about 4 to $6 \mathrm{keV}$. The photons significantly contributing to the dose extend to about $25 \mathrm{keV}$.

The fluence spectra were converted to dose equivalent using the corresponding conversion coefficients (ICRU, 1998; Behrens, 2017). The resulting dose rate depends on the processed material and its nature, varying from 1600 to 8300 , from 17 to $76 \mathrm{mSv} / \mathrm{h}$, and from 1 to $4 \mathrm{mSv} / \mathrm{h}$, for the quantities $\dot{H}^{\prime}(0,07), \dot{H}^{\prime}(3)$, and $\dot{H}^{*}(10)$, respectively. Such high dose rates would exceed legal dose limits within a few minutes to $1 \mathrm{~h}$ (for the local skin dose estimated by $\dot{H}^{\prime}(0,07)$ and the eye-lens dose estimated by $\dot{H}^{\prime}(3)$ ) or a few hours (for the effective dose of the whole body estimated by $\dot{H}^{*}(10)$ ).
Fortunately, in the normal case, the laser processing is performed in a laser protection housing, which is sufficient to absorb the photons. If, however, the laser intensity in materials processing rises in the future, the laser protection housing may no longer be sufficient to shield the photons.

\section{Conclusions}

The measurements performed, traceable to the Système international d'unités (SI), not only provide manufacturers and users of ultra-short pulsed lasers with important radiation protection information for the design of machines, but have also provided important input for recent legislative procedures in the field of radiation protection in Germany (StrlSchG, 2017; StrlSchV, 2018). Meanwhile, machines with even higher laser intensities are already under development. Therefore, the measuring method presented here will become even more relevant in the future - especially for cases in which laser intensities reach more than $10^{14} \mathrm{~W} / \mathrm{cm}^{2}$. Then, significantly higher energies and larger dose rates would be expected, resulting possibly in significant doses outside of the machine's housings and leading to radiation protection issues. 


\section{Appendix A: Details of the Bayesian spectrum deconvolution}

Equation (A1) represents a mathematical description of the experiment and thus the basic formula to be solved to obtain the fluence spectrum from the measured data:

$\boldsymbol{D}_{\text {meas }} \approx \mathbf{R} \cdot \boldsymbol{\Phi}=\boldsymbol{D}_{\text {calc }}$,

with measured doses $\boldsymbol{D}_{\text {meas }}=\left(\begin{array}{c}D_{\text {meas, } 1} \\ \vdots \\ D_{\text {meas }, N}\end{array}\right)$, calculated responses $\mathbf{R}=\left(\begin{array}{ccc}R_{1,1} & \cdots & R_{1, M} \\ \vdots & \ddots & \vdots \\ R_{N, 1} & \cdots & R_{N, M}\end{array}\right)$, and fluences $\boldsymbol{\Phi}=$ $\left(\begin{array}{c}\Phi_{1} \\ \vdots \\ \Phi_{M}\end{array}\right)$. The use of the symbol $\approx$ is meant to indicate that a strict equality is not achievable in practice due to uncertainties associated with the measurement. The analysis of the data was carried out using $N=13$ TLD layers. (The spectrometer consists of 30 TLD layers; however, significant doses above a few $\mu$ Gy were measured only in the first 13 layers, which, thus, provided the data points for the evaluation.) The fluence was binned into $M=60$ energy channels (with logarithmically increasing mean energy, as the energy resolution gets worse as the energy increases; 60 channels were chosen to cover the range from 2 to $100 \mathrm{keV}$ photon energy and to obtain the desired energy resolution of about $5 \mathrm{keV}$ at the upper limit).

The data, $D_{\text {meas }}$, and the response matrix, $\mathbf{R}$, are known: $\mathbf{R}$ was calculated using Monte Carlo radiation transport simulation package EGS4 (Nelson et al., 1985; Namito et al., 1995; Bielajew and Rogers, 1987), while the fluences, $\Phi(E)$, are determined from the Bayesian analysis.

$$
\Phi(E)=\left\{\begin{array}{c}
0 \text { for } E<E_{\text {rise, start }} \\
a_{\text {fluor }} \cdot \delta\left(E-E_{\text {fluor }}\right)+a_{\text {exp }} \cdot \exp \left(s_{\exp } \cdot E\right) \\
\cdot\left\{1+\cos \left[\left(E \cdot m_{\text {rise }}+b_{\text {rise }}\right) \cdot \pi\right]\right\} / 2 \\
\text { for } E_{\text {rise, start }}<E<E_{\text {rise, end }} \\
a_{\text {fluor }} \cdot \delta\left(E-E_{\text {fluor }}\right)+a_{\text {exp }} \cdot \exp \left(s_{\exp } \cdot E\right) \\
\text { for } E_{\text {rise, end }}<E<E_{\text {drop, start }} \\
a_{\text {fluor }} \cdot \delta\left(E-E_{\text {fluor }}\right)+a_{\text {exp }} \cdot \exp \left(s_{\exp } \cdot E\right) \\
\cdot\left\{1-\cos \left[\left(E \cdot m_{\text {drop }}+b_{\text {drop }}\right) \cdot \pi\right]\right\} / 2 \\
\text { for } E_{\text {drop, start }}<E<E_{\text {drop, end }} \\
0 \text { for } E_{\text {drop, end }}<E
\end{array}\right.
$$

$a_{\text {fluor }}$ is the amplitude of the fluorescence radiation, $E_{\text {fluor }}$ the energy of the fluorescence radiation, $a_{\exp }$ the amplitude of the exponential decay, $s_{\exp }$ the slope of the exponential decay, $m_{\text {rise }}=1 /\left(E_{\text {rise, end }}-E_{\text {rise, start }}\right), b_{\text {rise }}=$ $-m_{\text {rise }} \cdot E_{\text {rise, end }}, \quad m_{\text {drop }}=-1 /\left(E_{\text {drop, end }}-E_{\text {drop, start }}\right)$, $b_{\text {drop }}=-m_{\text {drop }} \cdot E_{\text {drop, end }}, E_{\text {rise, start }}$ the energy where the rise of the spectrum starts, $E_{\text {rise, end }}$ the energy where the spectrum turns into the exponential decay, $E_{\text {drop, start }}$ the energy where the drop of the spectrum starts, and $E_{\mathrm{drop}}$, end the energy where the spectrum drops to zero. In total, these are 12 parameters, of which 4 depend on other parameters $\left(m_{\text {rise }}, b_{\text {rise }}, m_{\text {drop }}\right.$, and $\left.b_{\text {drop }}\right)$, while $E_{\text {fluor }}$ is a constant (6.5 $\mathrm{keV}$ was chosen for targets made of steel, corresponding to the rounded mean energy of the $\mathrm{K}_{\alpha}$ and $\mathrm{K}_{\beta}$ emission lines from iron and $9 \mathrm{keV}$ for targets made of tungsten or the alloy containing more than $90 \%$ tungsten, corresponding to the rounded mean energy of the $L_{\alpha}$ and $L_{\beta}$ emission lines from tungsten). The remaining seven free parameters were determined from the data using a program written with the Bayesian software WinBUGS (Lunn et al., 2010). Appropriate initial values and ranges within which the optimization process was allowed to vary the parameters were chosen for the evaluation carried out by WinBUGS. 
Code availability. The WinBUGS code is available at https:// www.mrc-bsu.cam.ac.uk/software/bugs/the-bugs-project-winbugs/ (WinBUGS, 2021).

Data availability. The data are not available as they contain details from the laser machine's internal design and are therefore the property of the laser machine's owner, i.e. TRUMPF Laser GmbH.

Author contributions. RB contributed to the research plan, carried out the measurements, performed the data evaluation, and wrote the manuscript. BP contributed to the research plan and mainly performed the measurements. MR contributed to the data evaluation and the manuscript.

Competing interests. The authors declare that they have no conflict of interest.

Special issue statement. This article is part of the special issue "Sensors and Measurement Science International SMSI 2020". It is a result of the Sensor and Measurement Science International, Nuremberg, Germany, 22-25 June 2020.

Financial support. This research has been supported by TRUMPF Laser GmbH, Aichhalder Straße 39, 78713 Schramberg, Germany (grant no. 1W-63010 (2018-04-04)).

This open-access publication was funded by the Physikalisch-Technische Bundesanstalt.

Review statement. This paper was edited by Thomas Fröhlich and reviewed by two anonymous referees.

\section{References}

Ankerhold, U.: Catalogue of X-ray spectra and their characteristic data - ISO and DIN radiation qualities, therapy and diagnostic radiation qualities, unfiltered X-ray spectra, PTB report Dos-34, https://doi.org/10.7795/110.20190315B, 2000.

Ankerhold, U., Behrens, R., and Ambrosi, P.: X-ray spectrometry of low energy photons for determining conversion coefficients from air kerma, $K_{a}$, to personal dose equivalent, $H_{p}(10)$, for radiation qualities of the ISO narrow spectrum series, Radiat. Prot. Dosim., 81, 247 https://doi.org/10.1093/oxfordjournals.rpd.a032591, 1999.

Behrens, R.: A spectrometer for pulsed and continuous photon radiation, J. Instrum., 4, P03027, https://doi.org/10.1088/17480221/4/03/P03027, 2009.

Behrens, R.: Conversion coefficients for $H^{\prime}(3 ; \Omega)$ for photons, J. Radiol. Prot., 37, 354, https://doi.org/10.1088/1361-6498/aa51e8, 2017.

Behrens, R. and Ambrosi, P.: A TLD-based Few-channel Spectrometer for Mixed Photon, Electron, and Ion Fields with High Fluence Rates, Radiat. Prot. Dosim. 101, 73, https://doi.org/10.1093/oxfordjournals.rpd.a006062, 2002.

Behrens, R. and Reginatto, M.: Bayesian Spectrum Deconvolution Including Uncertainties and Model Selection: Application to XRay Emission Data Using WinBUGS, Radiat. Prot. Dosim., 185, 157-167, https://doi.org/10.1093/rpd/ncy286, 2019.

Behrens, R., Pullner, B., and Reginatto, M.: X-Ray Emission from Materials Processing Lasers, Radiat. Prot. Dosim. 183, 361-374, https://doi.org/10.1093/rpd/ncy126, 2019.

Bielajew, A. and Rogers, D. W. O.: PRESTA: The Parameter Reduced Electron-Step Transport Algorithm for Electron Monte Carlo Transport, Nucl. Instrum. Methods, 18, 165, https://doi.org/10.1016/S0168-583X(86)80027-1, 1987.

Gerhard, C., Viöl, W., and Wieneke, S.: Plasma-Enhanced Laser Materials Processing, Plasma Science and Technology, Progress in Physical States and Chemical Reactions, Tetsu Mieno, IntechOpen, London, available at: https://cdn.intechopen.com/pdfs/ 49531.pdf (last access: 16 February 2021), 2016.

International Commission on Radiation Units and Measurements (ICRU): Conversion Coefficients for use in Radiological Protection against External Radiation, ICRU Report 57, ICRU, Bethesda, https://doi.org/10.1093/jicru/os29.2.Report57, 1998.

International Organization for Standardization (ISO): Radiological protection, $\mathrm{X}$ and gamma reference radiation for calibrating dosemeters and doserate meters and for determining their response as a function of photon energy - Part 1: Radiation characteristics and production methods, ISO 4037-1, 2019.

Lunn, D. J., Thomas, A., Best, N., and Spiegelhalter, D.: WinBUGS - a Bayesian modelling framework: Concepts, structure, and extensibility, Stat. Comput., 10, 325, https://doi.org/10.1023/A:1008929526011, 2010.

Momma, C., Chichkova, B. N., Nolte, S., von Alvensleben, F., Tünnermann, A., Welling, H., and Wellegehausen, B.: Shortpulse laser ablation of solid targets, Opt. Commun., 129, 134, http://dx.doi.org/10.1016/0030-4018(96)00250-7, 1996.

Mulser, P. and Bauer, D.: High Power Laser-Matter Interaction, Springer, Berlin and Heidelberg, Germany, https://doi.org/10.1007/978-3-540-46065-7, 2010.

Namito, Y., Ban, S., and Hirayama, H.: LSCAT: Low-Energy Photon-Scattering Expansion for the EGS4 Code, National Laboratory for High Energy Physics, Report KEK Internal 95-10, 51 pp., Tsukuba, Japan, 1995.

Nelson, W. R., Hirayama, H., and Rogers, D. W. O.: The EGS4 Code System, Report SLAC-265, Stanford Linear Accelerator Center, 398 pp., Virginia, 1985.

Nolte, S., Schrempel, F., and Dausinger, F.: Ultrashort pulse laser technology, Laser sources and applications, Springer, Cham, Heidelberg, New York, Dordrecht, London, https://doi.org/10.1007/978-3-319-17659-8, 2016.

StrlSchG: Radiation Protection Act of 27 June 2017, (BGB1. 1966), as last amended by Article 5 of the Act of 23 October 2020 (BGB1. 2232), 2017.

StrlSchV: Radiation Protection Ordinance of 29 November 2018, (BGB1. 2034, 2036), as last amended by Article 1 of the Ordinance of 20 November 2020 (BGB1. 2502), 2018.

WinBUGS: WinBUGS code, University of Cambridge, MRC Biostatistics Unit, available at: https://www.mrc-bsu.cam.ac.uk/ software/bugs/the-bugs-project-winbugs/, last acess: 16 February 2021 . 